\title{
Mobile Monitoring of Traumatic Brain Injury in Older Adults: Challenges and Opportunities
}

\author{
Andrei Irimia ${ }^{1}\left(\mathbb{D} \cdot\right.$ Susan $_{\mathrm{Wei}^{2}} \cdot$ Nanshu $\mathrm{Lu}^{3} \cdot$ Constance M. Moore ${ }^{4}$. \\ David N. Kennedy ${ }^{5}$
}

Published online: 26 July 2017

(C) Springer Science+Business Media, LLC 2017

Keywords Mobile device $\cdot$ Neuroimaging .

Electroencephalography $\cdot$ Traumatic brain injury

Throughout the past decade, the use of mobile sensors to monitor human physiology has emerged as a promising strategy for encouraging healthy behaviors, assisting self-management of chronic disease, reducing health problems, decreasing the number of healthcare visits and facilitating beneficial interventions to improve well-being. ${ }^{1}$ Devices which facilitate periodic and/or continuous monitoring of key physiological parameters such as heart rate, blood pressure and glucose levels have been growing

\footnotetext{
${ }^{1}$ Kumar, S., Nilsen, W. J., Abernethy, A., Atienza, A., Patrick, K., Pavel, M., et al. (2013). Mobile health technology evaluation: the mHealth evidence workshop. American Journal of Preventive Medicine, 45(2), 228-236.
}

Andrei Irimia

irimia@usc.edu

1 Ethel Percy Andrus Gerontology Center, Leonard Davis School of Gerontology, University of Southern California, 3715 McClintock Avenue, Los Angeles, CA 90089, USA

2 Division of Biostatistics, School of Public Health, University of Minnesota, 420 Delaware Street SE, Minneapolis, MN 55455, USA

3 Department of Aerospace Engineering and Engineering Mechanics, Cockrell School of Engineering, University of Texas, 210 East 24th Street, Austin, TX 78705, USA

4 Center for Comparative Neuroimaging \& Department of Psychiatry, University of Massachusetts Medical School, 365 Plantation Street, Biotech One, Worcester, MA 01605, USA

5 Eunice Kennedy Shriver Center \& Department of Psychiatry, University of Massachusetts Medical School, 55 Lake Avenue North, Worcester, MA 01605, USA in popularity, and extensive efforts have been dedicated to the development of bioinformatic approaches for their automated analysis and interpretation. ${ }^{2}$

Older adults constitute a particularly suitable target population for mobile monitoring due to their greater susceptibility to disease, higher risk for complications following clinical interventions, reduced mobility, and numerous other reasons. ${ }^{3}$ For example, individuals over the age of 65 are considerably more susceptible to traumatic brain injury (TBI) than their younger counterparts partly because senior citizens have more limited motor ability and poorer physical balance. Similarly, many older adults live unassisted and are frequently unable to seek immediate medical care for their acute clinical needs following a TBI, leading to higher risks for sequelae. These factors increase the risk for the accelerated degradation of neurological function and for lasting vulnerability to co-morbidities. ${ }^{4}$ Even in relatively mild cases, the diminished ability of the aging brain to repair itself is paralleled by inadequate neuroimmune responses to injury, by greater susceptibility to adverse neurovascular events and by a higher risk for post-traumatic epileptogenesis. ${ }^{5}$ As a result, up to two thirds of ambulatory mTBI patients develop lasting neural and cognitive deficits whose onset might

\footnotetext{
${ }^{2}$ Zheng, Y. L., Ding, X. R., Poon, C. C., Lo, B. P., Zhang, H., Zhou, X. L., et al. (2014). Unobtrusive sensing and wearable devices for health informatics. IEEE Transactions on Biomedical Engineering, 61(5), 1538-1554.

${ }^{3}$ Ni Scanaill, C., Carew, S., Barralon, P., Noury, N., D, L., \& GM, L. (2006). A review of approaches to mobility telemonitoring of the elderly in their living environment. Annals of Biomedical Engineering, 34(4), 547-563.

${ }^{4}$ Harvey, L. A., \& Close, J. C. (2012). Traumatic brain injury in older adults: characteristics, causes and consequences. Injury, 43(11), 1821-1826.

${ }^{5}$ Stocchetti, N., Paterno, R., Citerio, G., Beretta, L., \& Colombo, A. (2012). Traumatic brain injury in an aging population. Journal of Neurotrauma, 29(6), $1119-1125$.
} 
be delayed or prevented if the risk for sequelae could be assessed on a patient-tailored basis early after injury. ${ }^{6}$

Two important goals of mTBI care involve understanding (1) how brain activity patterns change after trauma and (2) how such changes may reflect true injury severity and clinical outcome. Though significant, such goals remain elusive partly due to the difficulty, cost and logistic challenges of monitoring brain activity over extended periods of time. The overwhelming majority of mTBI patients are released from hospital on the day of injury, and the feasibility of comprehensive, indepth clinical follow-ups in the subacute stage of injury remains very limited. Because rigorous characterization of what happens to the concussed brain in the days and weeks after injury is often lacking, unraveling the effects of mTBI upon neurological health and upon the subsequent recovery or degradation of brain function remains problematic. Compounding these challenges are the high attrition rates of studies involving older victims of TBI. ${ }^{7}$

Though accelerometers have been used to detect falls in older adults, ${ }^{8}$ mobile solutions for brain wave monitoring and for their automatic analysis in ambulatory mTBI patients are currently lacking, as is the neuroinformatic infrastructure required to accommodate such solutions. Nevertheless, advances such as these could (1) improve TBI morbidity rates, (2) decrease health care costs, (3) guide clinical decisions on treatment type and aggressiveness, ${ }^{9,10}$ (4) facilitate the analysis and interpretation of neurophysiological signals acquired using mobile devices and (5) assist the process of obtaining novel insights into post-traumatic pathophysiology. Cloud computing, because of its ability to enable ubiquitous, ondemand, scalable processing of large datasets, is ideally positioned to assist mobile monitoring technologies, to streamline their analysis and to facilitate their interpretation.

Many electrophysiological monitoring methods target heart function because cardiac electrical activity and its pathophysiology are relatively well understood; for example, the electrocardiogram (ECG) is relatively easy to record and interpret in a clinical setting. By contrast, effective and affordable systems for monitoring brain activity are considerably

\footnotetext{
${ }^{6}$ De Beaumont, L., Theoret, H., Mongeon, D., Messier, J., Leclerc, S., Tremblay, S., et al. (2009). Brain function decline in healthy retired athletes who sustained their last sports concussion in early adulthood. Brain, 132, 695708.

${ }^{7}$ Corrigan, J. D., Harrison-Felix, C., Bogner, J., Dijkers, M., Terrill, M. S., \& Whiteneck, G. (2003). Systematic bias in traumatic brain injury outcome studies because of loss to follow-up. Archives of Physical Medicine and Rehabilitation, 84(2), 153-160.

${ }^{8}$ Lee, R. Y. W., \& Carlisle, A. J. (2011). Detection of falls using accelerometers and mobile phone technology. Age and Ageing, 40(6), 690-696.

${ }^{9}$ Chesnut, R. M. (1998). Implications of the guidelines for the management of severe head injury for the practicing neurosurgeon. Surgical Neurology, 50(3), 187-193.

${ }^{10}$ Watts, D. D., Hanfling, D., Waller, M. A., Gilmore, C., Fakhry, S. M., \& Trask, A. L. (2004). An evaluation of the use of guidelines in prehospital management of brain injury. Prehospital Emergency Care, 8(3), 254-261.
}

more challenging to design, implement, validate or commercialize. Electroencephalography (EEG) is by far the most common method for measuring brain activity non-invasively. This technique measures subtle voltage fluctuations (10 $100 \mu \mathrm{V}$ in amplitude) which occur across the scalp and are primarily due to the flow of ionic currents across the membranes of pyramidal neurons in the neocortex. ${ }^{11}$ Unlike electrocorticography (ECoG, which requires electrodes to be placed directly on the surface of the brain), EEG is widely regarded as a low-cost, portable, and non-invasive means to record brain activity and to monitor pathophysiology. Because the electroencephalogram (EEG) can be far more difficult to interpret, there has been far less progress toward the implementation of adequate informatic approaches for the management, analysis, curation and publication of data acquired using EEG systems. Nevertheless, mobile monitoring of brain function and the development of suitable strategies for on-demand analysis of brain signals using ubiquitous resources such as cloud computing should be prioritized particularly in the elderly, whose brains are at higher risk for neurological disturbances compared to other age groups.

Conventional EEG recordings are obtained by affixing individual, rigid electrodes onto the scalp using conductive gel after skin abrasion to reduce electrode-skin contact impedance. For decades, scalp EEG has suffered from limitations such as low spatial resolution, poor SNR, time-consuming and obstructive electrode connections, and short measurement times due to gel drying out. Additional disadvantages of conventional EEG involve the incompatibility between human skin - which is soft, curvilinear, and deformable - and the hard, planar, and rigid electrodes and electronics. The electrode cables of conventional EEG systems encumber patients and their movement produces motion artefacts which degrade signal quality and which render longterm data acquisition both challenging and impractical. For reasons such as these, future sensor arrays and bioinformatics solutions for EEG should incorporate state-of-the-art advances in electrode design and wireless technology. One solution to this could involve the integration of EEG with an epidermal electrode system (EES); this increasingly-popular technology involves a scalable array of low-cost, fully-wireless, epidermal, bipolar EEG sensors equipped with miniaturized printed circuit boards (PCBs) for long-term, high-fidelity data acquisition and wireless transmission to mobile phones. ${ }^{12,13}$ Because skin sensitivity and susceptibility to bruises increases with age, such a system could

\footnotetext{
${ }^{11}$ Irimia, A., Van Horn, J. D., \& Halgren, E. (2012). Source cancellation profiles of electroencephalography and magnetoencephalography. Neuroimage, 59(3), 2464-2474.

${ }^{12}$ Kim, D. H., Lu, N., Ma, R., Kim, Y. S., Kim, R. H., Wang, S., et al. (2011). Epidermal electronics. Science, 333(6044), 838-843.

${ }^{13}$ Siegel, A. C., Phillips, S. T., Dickey, M. D., Lu, N. S., Suo, Z. G., \& Whitesides, G. M. (2010). Foldable Printed Circuit Boards on Paper Substrates. Advanced Functional Materials, 20(1), 28-35.
} 
be more adequate for older adults because it can greatly reduce the risk of epidermal irritation and scarring.

Though most applications of EEG involve the acquisition and analysis of sensor-space brain wave signals, the inverse localization of neural electrical activity can be of additional use because it allows the electrical currents which generate the EEG to be mapped on the cortical surface. This can provide valuable neuroanatomic localization, with important consequences for the ability to identify brain regions at risk for concussion-related sequelae such as atrophy, loss of neuronal connectivity and pathophysiology. ${ }^{14,15,16}$ Thus, instead of implementing informatics solutions which limit themselves to facilitating temporal analysis of EEG voltages, future information processing streams should aim to accommodate spatiotemporal analysis of electrical currents which are inversely mapped over the surface of the brain. ${ }^{17}$ Such an approach is much more powerful than simply analyzing EEG traces because it can facilitate the identification of specific gyri and sulci where electrical activity originates. This can allow clinicians to determine which cortical structures are affected by pathophysiology and then to identify specific brain functions which are localized in these structures and which could be at high risk for decline after mTBI.

A key feature of acute mTBI pathophysiology is that much of it can be transient, meaning that abnormal brain waves which are indicative of future functional decline can occur spontaneously and relatively infrequently. ${ }^{18}$ This implies that EEG monitoring of mTBI patients may need to be performed continuously over the span of several hours, days or even weeks. As previously suggested, however, monitoring brain activity in ambulatory patients over extended periods of time is associated with substantial challenges related to the recording, wireless transmission, storage, archiving, consolidation, classification, analysis and interpretation of large and information-rich spatiotemporal series. Continuous EEG monitoring can thus easily become a prohibitively-difficult big data problem which cloud computing is ideally positioned to address. When confronted with temporally-long datasets,

\footnotetext{
${ }^{14}$ Irimia, A., Goh, S. Y., Torgerson, C. M., Chambers, M. C., Kikinis, R., \& Van Horn, J. D. (2013). Forward and inverse electroencephalographic modeling in health and in acute traumatic brain injury. Clinical Neurophysiology, 124(11), 2129-2145.

${ }^{15}$ Irimia, A., Goh, S. Y., Torgerson, C. M., Stein, N. R., Chambers, M. C., Vespa, P. M., et al. (2013). Electroencephalographic inverse localization of brain activity in acute traumatic brain injury as a guide to surgery, monitoring and treatment. Clinical Neurology \& Neurosurgery, 115(10), 2159-2165.

${ }^{16}$ Irimia, A., \& Van Horn, J. D. (2015). Epileptogenic focus localization in treatment-resistant post-traumatic epilepsy. Journal of Clinical Neuroscience, 22(4), 627-631.

17 Salman, A., Malony, A., Turvets, S., Volkov, V., Ozog, D., \& Tucker, D. (2016). Concurrency in electrical neuroinformatics: parallel computation for studying the volume conduction of brain electrical fields in human head tissues. Concurrency and Computation, 28(7), 2213-2236.

${ }^{18}$ Werner, C., \& Engelhard, K. (2007). Pathophysiology of traumatic brain injury. British Journal of Anaesthesiology, 99(1), 4-9.
}

the clinical practice of inspecting raw EEG traces visually to identify neuropathophysiology becomes prohibitively timeconsuming and, ultimately, unfeasible. Consequently, automated methods for identifying abnormal activity in such data are greatly needed, as are informatics methodologies for the archival, curation and rapid analysis of large neurophysiological datasets acquired from ambulatory patients. Because of the temporal length and informational complexity of EEG time series acquired continuously using mobile devices, advanced methods for time series analysis need to be developed and incorporated into the architecture of neuroinformatics systems to identify abnormal brain activity automatically. Such big data science solutions should be scalable as a function of acquisition time and cohort size, they must be computationally efficient, cost-effective and compatible with clinicians' needs and expectations.

The development of next-generation EEG neuroinformatics will likely require new ways to conceptualize big data, to extract meaningful information from it and to synthesize this information in clinically-useful ways. Recent advances in machine learning (ML) and topological data analysis (TDA) can likely provide the much-needed ability to analyze very large datasets of neurophysiological recordings and to identify transient pathophysiology in high-dimensional, high-velocity spatiotemporal datasets of neurophysiological origin. The development of ML and TDA approaches which are task-driven, ondemand or on-line could address critical gaps in existing methods for the classification of high-velocity data streams. Particularly novel and innovative are joint learning approaches to feature representation and classification of spatiotemporal data series because such methods have offered higher reproducibility than conventional methods in fields as varied as image segmentation ${ }^{19}$ and gene expression classification. ${ }^{20}$

EEG and EEG-derived data are strongly temporal or even spatiotemporal in the case of inversely-localized neurophysiological signals. Because these properties can benefit our understanding of neurobiology and neuropathophysiology, the structure and topology which are intrinsic to such data should be leveraged rather than ignored. The application of ML to EEG high-velocity data streams could lead to the development of entirely new branches of ML, and this could have substantial impact not only upon mobile health data analytics but also upon ML in general. While such innovations could benefit the state of the art in the informatics and analysis of neurophysiological signals, there is an even broader set of applications which could benefit from these methodologies. Specifically,

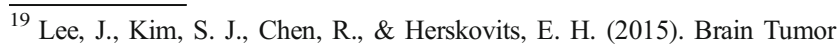
Image Segmentation Using Kernel Dictionary Learning. 37th Annual International Conference of the IEEE Engineering in Medicine and Biology Society, 658-661.

${ }^{20}$ Tian, Z., Hwang, T., \& Kuang, R. (2009). A hypergraph-based learning algorithm for classifying gene expression and arrayCGH data with prior knowledge. Bioinformatics, 25(21), 2831-2838.
} 
electrophysiological data are typical of the characteristics exhibited by data streams measured in a wide variety of mobile health devices, since these data are not only (A) large from the standpoint of their space complexity, but also (B) high in velocity, meaning that data are collected rapidly in real time. Thus, the development of techniques for analyzing this type of mobile-health data is appealing not only in the context of our own area of interest, but may also offer viable options for realizing the true potential for on-demand analysis of large datasets with temporally- and spatially-informative properties. Incidentally, prior informatics efforts indicate that cloud computing is well suited for the compression and subsequent analysis of large spatiotemporal datasets. ${ }^{21}$

An acute challenge of contemporary neurophysiological data science and bioinformatics is that the datasets involved are often voluminous, structurally complex, high-dimensional and noisy, both stochastically and deterministically. TDAwhose potential and value to data scientists has started to be appreciated only within the past several years - is ideally suited for processing such data because this family of analysis methods can identify significant data clusters hierarchically and can allow one to apply a divide-and-conquer approach to big data analysis. ${ }^{22}$ TDA can identify prominent geometric features, infer local and global properties and explore data properties in high dimensions without the absolute need for dimensionality reduction. Understanding data topology can substantially assist supervised learning, particularly for timeseries anomaly detection from EEG. These advantages are likely to be substantial when analyzing the post-traumatic EEG of older adults due to the potential complexity of anomalous electrophysiological manifestations observed in this high-risk cohort. ${ }^{23}$

In conclusion, thanks to recent advances in mobile sensor technology and spatiotemporal data analysis, neuroinformaticians are now well positioned to lead the way in formulating effective approaches to assist the task of monitoring brain activity using wireless devices and cloud computing. Whereas such monitoring could, in principle, be useful to a substantial cross-section of the TBI patient population, older victims of mTBI are particularly likely to benefit from this type of technology due to their greater vulnerability to functional decline after mTBI. Future efforts should be dedicated to the design and implementation of efficient neuroinformatics approaches which can facilitate the classification of EEG data and, by extension, that of large, information-rich spatiotemporal series. Because current solutions to this challenging problem lag very much behind our current ability to collect high-velocity, long-duration data streams, advances in this area of informatics would fill a critical gap in the array of methods which are necessary for continuously monitoring the brain activity of mTBI patients and for clinical neuroelectrophysiology in general. When assisted by cloud computing, the information technology which could result from such efforts would offer promising new ways to leverage the richness of biomedical big data for personalized neurology.

Acknowledgments Part of the research leading to the insights expressed in this editorial was made possible through the generous support of the National Institute of Environmental Health Sciences (NIEHS) via the Training Coordination Center (TCC) of the Big Data to Knowledge (BD2K) Program (bigdatau.ini.usc.edu). As such, part of this work was supported by the National Institutes of Health (NIH), grant U24 ES 026465 to Prof. John D. Van Horn. The TCC is hosted by the USC Mark \& Mary Stevens Neuroimaging and Informatics Institute at the University of Southern California. The collaboration between co-authors was facilitated by the TCC via its 2016 Innovation Lab on Mobile Health and its 2017 Data Science Rotations for Advancing Discovery (RoAD) Trip program. This editorial was written while A. I. was a RoAD Trip Fellow in the Department of Psychiatry at the University of Massachusetts Medical School, where D. N. K. hosted and mentored him. A. I. is also supported by NIH grant R01 NS 100973. D. N. K. is supported, in part, by NIH grants P41 EB 019936, R01 MH 083320 and R43 NS 074540 . The authors wish to personally thank Prof. John D. Van Horn and Ms. Crystal Stewart. The content of this editorial is solely the responsibility of the authors and does not necessarily represent the official views of the NIH.

\section{Compliance with Ethical Standards}

Conflict of Interest The authors declare that they have no conflict of interest.

\footnotetext{
${ }^{21}$ Yang, C., Zhang, X. Y., Zhong, C. M., Liu, C., Pei, J., Ramamohanarao, K., et al. (2014). A spatiotemporal compression based approach for efficient big data processing on Cloud. Journal of Computer and System Sciences, 80(8), $1563-1583$.

${ }^{22}$ Pereira, C. M. M., \& de Mello, R. F. (2015). Persistent homology for time series and spatial data clustering. Expert Systems with Applications, 42(1516), 6026-6038.

${ }^{23}$ Jiang, L., Yin, X. H., Yin, C., Zhou, S. A., Dan, W \& Sun, X. C. (2011). Different quantitative EEG alterations induced by TBI among patients with different APOE genotypes. Neuroscience Letters, 505(2), 160-164.
} 